\title{
GLSCH: OBSERVATION SCHEDULER FOR THE GLORIA TELESCOPE NETWORK
}

\author{
C. López-Casado ${ }^{1}$, C. J. Pérez del Pulgar ${ }^{1}$, E. Fernández ${ }^{2}$, V. F. Muñoz ${ }^{1}$, and A. Castro-Tirado²
}

\begin{abstract}
This paper proposes the design and development of a scheduler for the GLORIA telescope network. This network, which main objective is to make astronomy closer to citizens in general, is formed by 18 telescopes spread over four continents and both hemispheres. Part of the management of this network is made by the network scheduler. It receives the observation requests made by the GLORIA users and then sends it to the most suitable telescope. A key module of the network scheduler is the telescope decision algorithm that makes possible to choose the best telescope, and thus avoiding offering an observation to a telescope that cannot execute it. This paper shows two different telescope decision algorithms: the first one is only based on weather forecast, meanwhile the second one uses fuzzy logic and information from each network telescope. Both algorithms were deployed in the GLORIA network. The achieved results coupled with a comparative of their performance is shown. Moreover, the network scheduler architecture, based on a hybrid distributed-centralized schema, is detailed.
\end{abstract}

\section{RESUMEN}

Este artículo presenta el diseño e implementación de un planificador de observaciones para la red de telescopios GLORIA. Esta red tiene como objetivo principal hacer que la astronomía sea más cercana a los ciudadanos. Está compuesta por 18 telescopios ubicados en cuatro continentes en ambos hemisferios. Parte de la gestión de esta red es llevada a cabo por el planificador. Éste se encarga de la recepción de las peticiones de observación realizadas por los usuarios de la red GLORIA y posteriormente decide el telescopio que realizará dicha observación. Un módulo clave dentro de este planificador es el algoritmo de decisión del telescopio que finalmente realizará la observación. Este artículo presenta dos tipos de algoritmos distintos: uno basado únicamente en la predicción meteorológica y otro basado en lógica difusa. Ambos algoritmos fueron desplegados en la red GLORIA. Los resultados y una comparativa entre ambos son presentados. Además, se detalla la arquitectura del planificador, basada en un esquema híbrido distribuido-centralizado.

Key Words: techniques: miscellaneous - telescopes

\section{INTRODUCTION}

Over the last decades, there has been an increase in telescope network implementations. There is a huge interest in making astronomy closer to students and citizens in general (Castro-Tirado et al. 2014; Gresham et al. 2016); providing easy-to-use web tools to awake their interest. On the other hand, telescope networks focused on professional astronomers have also been developed (Bakos \& Gaspar 2016; Bigongiari \& Consortium 2016).

Main part of these networks is based on a coordinator or scheduler that manages all the requests. These requests will include information about the target to observe and also how to observe it, i.e. the

\footnotetext{
${ }^{1}$ Departamento de Ingeniería de Sistemas y Automática (Unidad Asociada de I+D+i al CSIC), Universidad de Málaga, Escuela de Ingenierías Industriales. C/ Doctor Ortiz Ramos s/n., 29071 Málaga, España.

${ }^{2}$ Instituto de Astrofísica de Andalucía (IAA-CSIC), Glorieta de la Astronomía s/n, 18008 Granada, España.
}

constraints. Depending on this data, the scheduler will propose the observation to a specific telescope and afterwards, it will execute the observation itself. The design and implementation of the scheduler in the network can be distributed or fully centralized. The distributed architecture (Racero et al. 2015) usually includes a central node that decide the telescope and local nodes that schedule all the received observations. On the other hand, centralized systems (Saunders et al. 2014) schedule all the telescope observations in the central node. The telescope only acts as a sequencer, receiving instructions and generating data.

In the last term of 2014, the GLORIA network (Castro-Tirado et al. 2014) was launched with the aim of create a world-wide telescope network where users could research in astronomy using robotic telescopes, and/or analyzing data which other users have acquired. It integrates telescopes that have been 


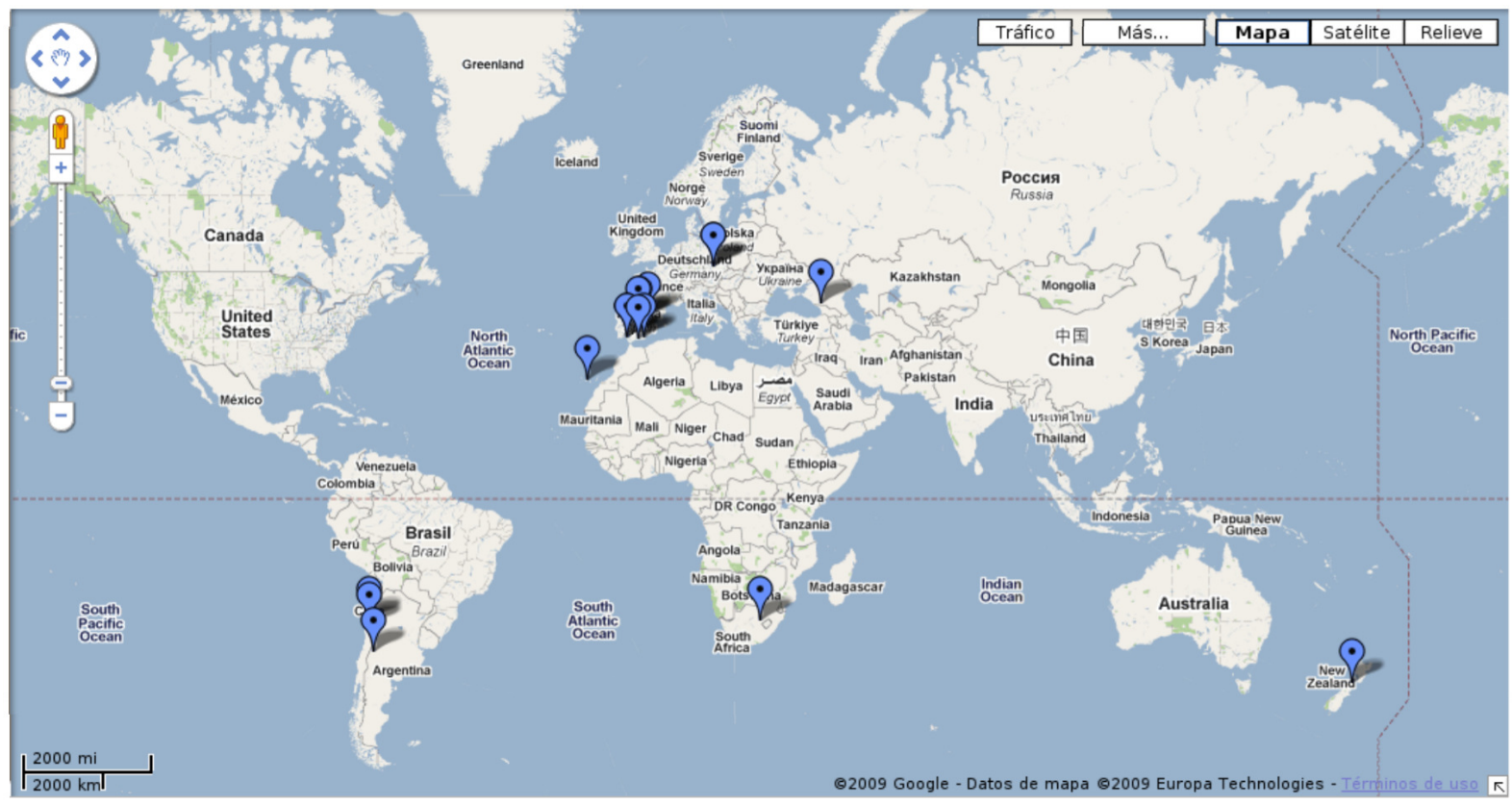

Fig. 1. Geographic location of the GLORIA telescopes.

working in various scientific fields and dissemination issues, creating a heterogeneous telescope network. As a part of this network, a scheduler has been designed and it is being implemented and deployed. This scheduler manages all the observation requests and decide the telescope that will be offered the observation. This decision is made through a telescope decision algorithm.

This paper details the GLORIA network in $\S 2$, as well as the architecture of the scheduler in $\S 3$. The different telescope decision algorithms are explained in $\S 4$. Next, the statistic results over the network usage are shown in $\S 5$. Finally, conclusions are in $\S 6$.

\section{THE GLORIA TELESCOPE NETWORK}

The GLORIA network integrates 18 telescopes worldwide (Figure 1) that had been working in various scientific fields and dissemination issues. Each telescope has its own different features and its own control system what make the network to be heterogeneous, both in optic characteristics and in telescope control systems. As all the telescope are already working in different issues, the telescope time is shared between owners and the GLORIA network.

The underlying idea in GLORIA is that "the more eyes we would put on the sky the more and greater the scientific discoveries that will be achieved". Taking advantage of collective intelli- gence (Wang et al. 2010) the huge amount of astronomical data can be analyzed by both professional and amateur astronomers. Thus, in order to try to improve the way of doing astronomy research, users will be guided through the different tasks the research requires. Each kind of research has been called experiment. There are two kinds of experiments: those that require a telescope, which have been called on-line experiments and off-line experiments which work on data produced by the GLORIA network. The available off-line experiments are: Personal Space, where you choose an important event in your life and see the sky that was directly overhead at that exact moment; or Solar Activity, where you can contribute to the measurement of solar activity; and Variable Stars that allows the analysis of the light curves of the stars.

On the other side, on-line experiments are also divided into two categories: teleoperation and batch. The first one is used to directly control the telescope at a specific date and time, which is chosen by the user. At that date, user will be able to control the telescope mount to point at a specific target, select the filter, focus and set the exposure time to take an image that can be finally downloaded. On the other hand, in batch mode, users do not control the telescope; instead, a request to observe a target is submitted to the network and the scheduler will manage the observations. 


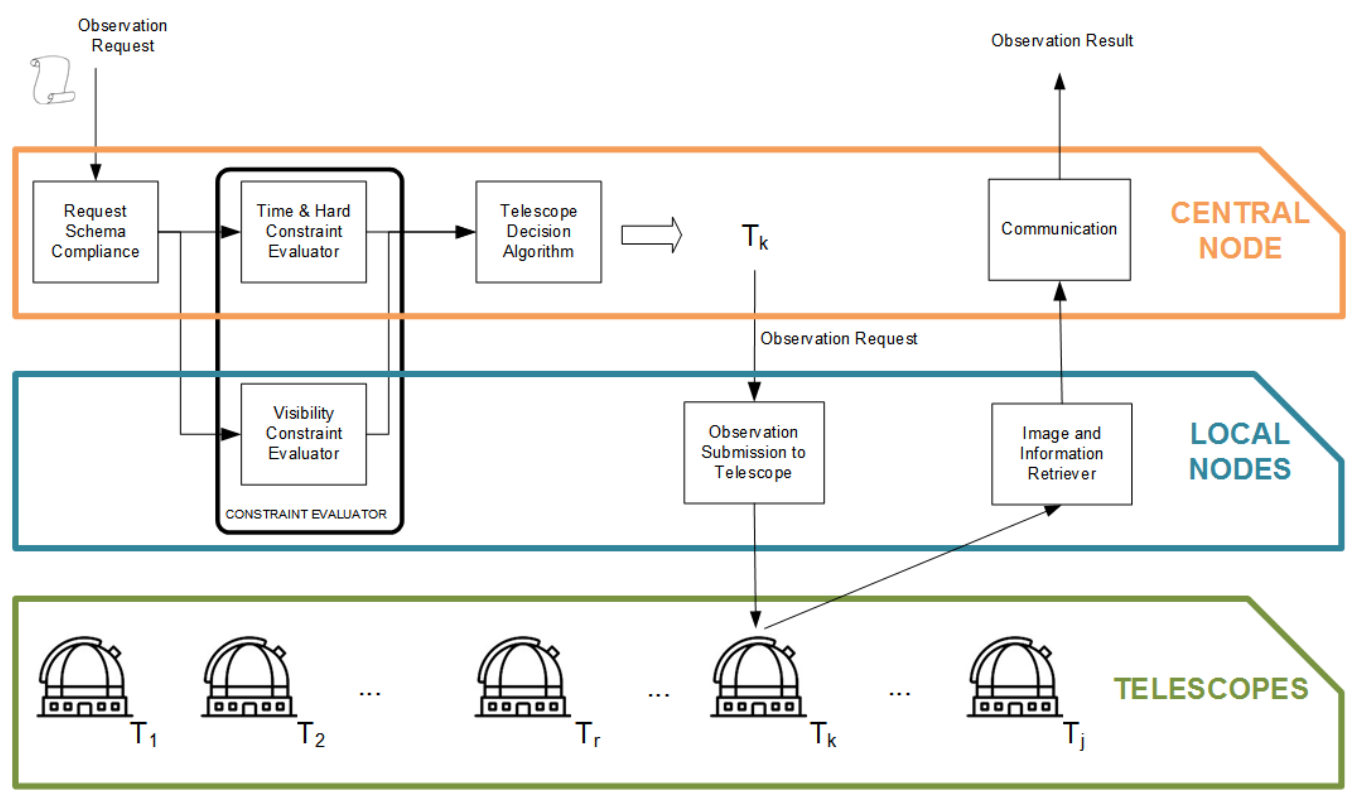

Fig. 2. GlSch architecture.

\section{GLSCH: OBSERVATION SCHEDULER}

Most of the telescope networks that are currently in use or that are being developed have a common characteristic: their telescopes are only used by the network and all of them have the same control system. However, one of the key factors of the GLORIA network is that the telescope usage in the network is shared with the telescope owner; the idea is to offer, at least, the telescope idle time to the GLORIA community. This private and public coexistence make mandatory the use of the telescope own control system and the local scheduler provided with it. This fact makes the GLORIA network to be heterogeneous not only because its telescopes have different features, but also because there are different control systems to manage them (RTS2 (Kubánek 2016), ACP (Denny 2011) ...). Moreover, to combine the existence of a network scheduler to manage the entire user requests, and also keep the telescope autonomy, the telescope local scheduler has to belong to the GLORIA scheduler architecture.

The GLORIA scheduler manages all the user requests, taking into account the constraints to be satisfied and deciding to which telescope the observation will be offered. Then, the own telescope scheduler will include the observation within the night plan which has to be executed. Figure 2 shows the scheduler hybrid distributed-centralised architecture. It is based on 3 layers: the upper one formed by a unique node, the Central Node. The middle layer where there is a Local Node for each telescope in the network and finally the lower layer where the telescopes themselves are located.

\subsection{Observation management process}

The first step of the observation management process is made by the Central Node, once the user has submitted the observation it has to assure that the request is compliant with the request schema. Then a constraint analysis is made. The constraints are divided into time constraints, hard constraints and visibility constraints. The analysis of the first two ones is made by the Central Node. Time constraints define a time window where the observation has to be performed. If this window is within the margins the scheduler is processing, the request analysis will continue; if not, it will be scheduled later. Next constraints to be checked are the hard ones; these are constant or can be considered as constant from the point of view of the telescope/observatory. The visibility constraints depend on the exact time the target will be observed, e.g. target altitude. The Central Node will communicate with the local nodes to provide them all the information needed to make a visibility study and decide if the target can be observed or not. Finally, the Central Node, through the decision algorithm, will choose the telescope that will be offered the observation.

The Local Node, associated to the chosen telescope, notifies the observation to the telescope local scheduler. The ways of submitting a new observation to the third-party schedulers includes from creating and uploading a new file (ACP) to use an specific in- 


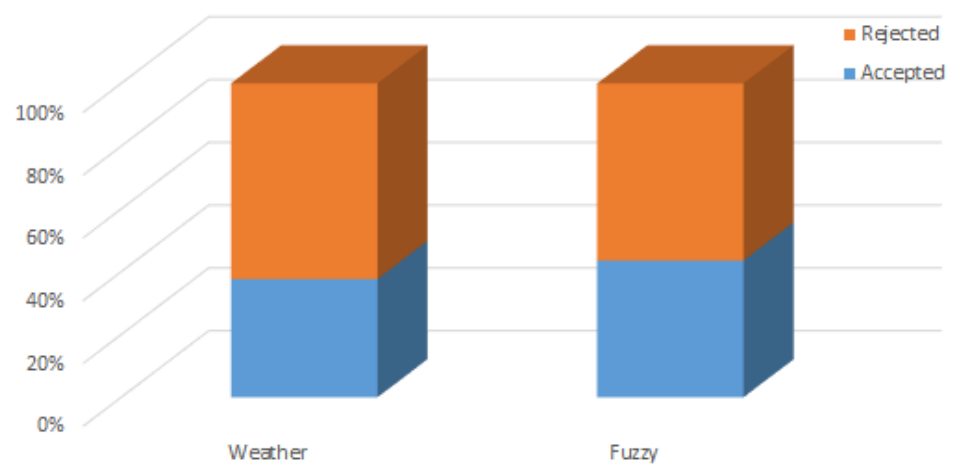

(a) Acceptance Rate

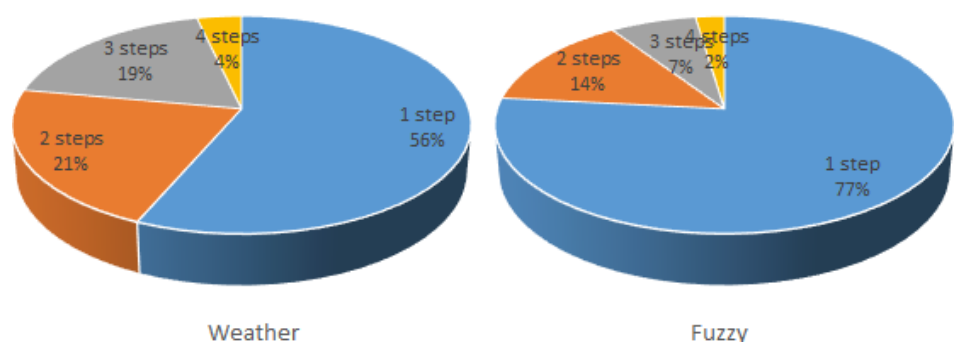

(b) Reallocation steps

Fig. 3. Algorithm performance comparative.

terface to create and then scheduler a target (RTS2, some custom systems...). Then the telescope scheduler manages the observation including it into its night plan. The result of this process can be positive when the observation images have been taken; or negative, when the observation has not been executed. If local telescope scheduler completes the observation, the local node fetches the images and a notification is sent to the central node which updates the request state.

On the other hand, when no images have been taken, a reallocation process, to choose another telescope, is started. This process is made by the Central Node that selects another telescope that will be offered the observation request. This process is repeated up to 5 times.

\section{TELESCOPE DECISION ALGORITHM}

The difference in the architecture and usage of the GLORIA network, in relation to the other telescope networks, makes the scheduler main goal to be also different. While minimizing the time per observation is the main objective in most of the telescope network, the principal GLORIA objective is to maximize the number of completed observations. As each GLORIA telescope has its own control system with its own local scheduler, the observation time cannot be controlled. Each GLORIA request will be included in the telescope night plan and GLORIA does not have any control about the execution time slot. To avoid offering an observation to a telescope that cannot execute it and then making the overall process longer, the telescope decision algorithm is crucial.

Next, the two telescope decision algorithms that have been designed, implemented and deployed in the GLORIA network are explained.

\subsection{Weather forecast algorithm}

This telescope decision algorithm is based on weather forecast. This information has been taken from 7timer (Ye 2011), a free project that provides specific information for astronomy. The telescope decision algorithm receives the weather forecast for every telescope that passed the constraints. For each of them, a score is computed taking into account the weather condition in its night hours, the parameters considered are the weather forecast (cloud cover and precipitation type) and the seeing.

\subsection{Fuzzy logic algorithm}

This telescope decision algorithm uses a fuzzy logic model to score each available telescope that passed the constraints. The fuzzy model uses different kind of input parameters: astronomical weather 
information, target quality observation and telescope network feedback.

The astronomical weather parameters informs about the weather condition at the observatory location: weather forecast and seeing, as they were used in the weather forecast algorithm, are used. The parameter used to measure the target quality at the observatory location is the Target Transit Altitude, i.e. the maximum altitude that the target can reach from the observatory location. Finally, there are two telescope network feedback parameters to include information about the previous performance of the telescopes. On one hand, the User Score, once an observation is completed, GLORIA users can score the image taken by the telescope. And in the other hand, the Telescope Acceptance Rate, the percentage of executed observations per telescope over the total offered ones.

\section{RESULTS}

The scheduler that has been described coupled with the detailed decision algorithms were deployed and tested into the GLORIA network. The experiment consisted of analyzing the information produced by the network during two periods. In the first one, the weather forecast algorithm was used, meanwhile in the second period the fuzzy logic one was active.

Figure 3a reveals that the acceptance rate for both type of algorithm are quite similar: 37,96\% when the weather forecast algorithm was in used and $43,58 \%$ when the fuzzy logic one was active. However, a deeper analysis on completed observations (Figure 3b), specifically in the number of reallocation steps, shows that when fuzzy logic algorithm is used the $77 \%$ of the completed observation were made by the first telescope the algorithm chose versus the $56 \%$ associated to the weather based algorithm. That means that the overall observation time were reduced when the fuzzy logic algorithm was used, exactly it was moved from 41 hours to less that 28 .

\section{CONCLUSIONS}

This paper has described the hybrid distributedcentralised architecture of the GLORIA scheduler, as well as the two different telescope decision algorithms that has been implemented and tested in the GLORIA network. One of them based on weather forecast and the other one based on fuzzy logic including different input parameters.

A comparative between the results of both algorithms in the GLORIA network has been made. This comparative shows that the fuzzy logic algorithm reduces the reallocation process and thus the overall time to complete the observations submitted by users.

Acknowledgments: This work has been partially supported by the GLORIA project. GLObal Robotic telescopes Intelligent Array for e-Science (GLORIA) is a project funded by the European Union Seventh Framework Programme (FP7/20072012) under grant agreement number 283783.

\section{REFERENCES}

Bakos, G. 2016, NASA Proposal \#16-XRP16, 70

Bigongiari, C. \& Consortium, C. 2016, NPPP, 279, 174

Castro-Tirado, A. J., Sánchez Moreno, F. M., Pérez del Pulgar, et al. 2014, RMxAA, 45, 104

Denny, R. 2011, Telescopes from Afar Conference, 47

Gresham, K. C., Palma, C., Polsgrove, D. E., et al. 2016, AcAau, 129, 130

Kubánek, P. 2016, SPIE, 9913, 2

Racero, E., Ocaña, F., \& Ponz, D. 2015, Highlights of Spanish Astrophysics, 828

Saunders, E. S., Lampoudi, S., Lister, T. A., Norbury, M., \& Walker, Z. 2014, SPIE, 9149, 0ES

Wang, F., Deng, H., Guo, L., \& Ji, K. 2010, in 2010 International Forum on Information Technology and Applications (IEEE), 417

Ye, Q.-Z. 2011, PASP, 123, 113 